\title{
REGISTRO DE IDENTIDADES DE ECOSISTEMAS SONOROS URBANOS COMO BASE PARA LA CREACIÓN ARTÍSTICA CONTEMPORÁNEA
}

\section{Jaime Cornelio Yacaman}

Investigador independiente

\section{Resumen}

La identidad sonora de un ecosistema se manifiesta de modo complejo, en constante transformación, respondiendo en cada momento a distintos fenómenos que constituyen o emergen en cada lugar. El registro de los sonidos que emergen de ecosistemas urbanos forma parte de una experiencia cognoscitiva que es posible analizar, identificar y usar en la creación plástica. A partir de las preguntas de investigación: ¿Qué elementos temporales, espaciales, geográficos, sociales y perceptivos (subjetivos) intervienen en la construcción de un ecosistema sonoro?, ¿Qué factores deben tomarse en consideración durante su registro?, y, ¿Cómo puede el registro de un sonido de un ecosistema indicarnos algo sobre la identidad de este ecosistema? El objetivo del artículo es analizar las relaciones entre sonido, música, arte y sociedad en la conformación de la identidad sonora territorial (ecosistemas sonoros) en algunos casos significativos. El análisis será realizado a partir de las teorías de la complejidad (Morin 2010; Whitehead 1920; Stengers 2005; Latour 1995; De Landa 1997) y algunos estudios de caso (obra propia; Vigueras 2016; Philipsz 2010; Geometry Global 2016; Heuson 2014). Este artículo pretende contribuir con una profundización del concepto de registro sonoro que puede servir a la creación de arte sonoro.

Palabras clave: IDENTIDAD; SONIDO; MÚSICA; ARTE; SOCIEDAD

\section{RECORDING THE IDENTITIES OF URBAN SOUND ECOSYSTEMS AS A BASIS FOR CONTEMPORARY ARTISTIC CREATION}

\section{Abstract}

The sound identity of an ecosystem manifests itself in a complex way, in constant transformation, responding at each moment to different phenomena that constitute or emerge in each place. Recording the sounds that emerge from urban ecosystems is part of a cognitive experience that can be analysed, identified and used in artistic creation. Based on the research questions: What temporal, spatial, geographical, social and perceptual (subjective) elements are involved in the construction of a sound ecosystem, what factors must be taken into consideration during its recording, and how can the recording of a sound from an ecosystem tell us something about the identity of this ecosystem? The aim of the article is to analyse the relationships between sound, music, art and society in the shaping of territorial sound identity (sound ecosystems) in some significant cases. The analysis will be carried out on the basis of complexity theories (Morin 2010; Whitehead 1920; Stengers 2005; Latour 1995; De Landa 1997) and some case studies (own work; Vigueras 2016; Philipsz 2010; Geometry Global 2016; Heuson 2014). This article aims to contribute to a deepening of the concept of sound recording that can serve the creation of sound art.

Keywords: IDENTITY; SOUND; MUSIC; ART; SOCIETY

\footnotetext{
Cornelio Yacaman, Jaime. 2021. "Registro de identidades de ecosistemas sonoros urbanos como base para la creación artística contemporánea". AusArt 9 (1): 143-157. DOI: 10.1387/ausart.22617
}

\section{AUSART}




\section{INTRODUCCIÓN}

La identificación y registro de los elementos que configuran la identidad sonora de cada ecosistema sonoro y su exploración artística son los focos de este artículo, y de la tesis de doctorado que desarrollo en la Universidad del País Vasco. Esta identificación y registro de elementos es compleja y no está libre de dificultades, especialmente para la práctica artística. El problema de investigación gira alrededor de cómo identificar los elementos que constituyen la identidad sonora del ecosistema desde el marco conceptual de la complejidad. En torno a este desafío y problema de investigación construyo las siguientes preguntas: ¿Qué es la identidad sonora de un lugar? ¿Qué elementos temporales, espaciales, geográficos, sociales y perceptivos (subjetivos) intervienen en la construcción de un ecosistema sonoro? ¿Qué factores tomar en consideración durante su registro? ¿Cómo puede el registro de un sonido de un ecosistema indicarnos algo sobre la identidad de este ecosistema?

Esto implica, por un lado, definir los conceptos de identidad sonora y ecosistema sonoro y, por otro, comprender los desafíos de carácter metodológico y técnico que la perspectiva compleja plantea para registrar un ecosistema sonoro. En este sentido, sugiero la hipótesis de que la identidad sonora de un ecosistema se manifiesta de modo complejo, en constante transformación, respondiendo en cada momento a distintos fenómenos que constituyen o emergen en cada lugar. Los distintos tipos de factores que deben tomarse en consideración a la hora de registrar la identidad sonora de un lugar son geológicos, territoriales, meteorológicos, temporales, espaciales, sociales, psicológicos y físicos. Entre estos elementos, el registro de sonidos urbanos supone una serie de relaciones que se establecen entre sonido, música, arte y sociedad. Las identidades sonoras de cada lugar provocan experiencias perceptivas y cognoscitivas que pueden ser identificadas y analizadas desde el paradigma de las teorías de la complejidad.

Según la Real Academia Española (RAE) un ecosistema es la "comunidad de los seres vivos cuyos procesos vitales se relacionan entre sí y se desarrollan en función de los factores físicos de un mismo ambiente"1. El concepto de ecosistema entendido desde las teorías de la complejidad está en la base del enfoque de esta investigación. Este concepto puede expresarse, ampliando la definición de la RAE, como una comunidad de los seres vivos y no vivos (naturales y artificiales), cuyos procesos vitales se relacionan entre sí y se desarrollan en función de los factores físicos, sociales, económicos, históricos, y simbólicos de un mismo ambiente manteniendo una constante relación entre 
orden-desorden-organización, a partir de las interacciones que se producen y que condicionan el medio. Estas interacciones son identificadas como las acciones que modifican el comportamiento o la naturaleza de los elementos que intervienen e interactúan en un tiempo y lugar determinado.

Relacionando el concepto de ecosistema con el sonido, la identidad y territorio sugerimos el concepto de ecosistema sonoro. A partir del concepto de ecosistema sonoro el objetivo de este artículo es analizar las relaciones entre sonido, música, arte y sociedad en la conformación de la identidad sonora territorial (ecosistemas sonoros) en algunos casos significativos. El análisis será realizado a partir de las teorías de la complejidad (Morin 2010; Whitehead 1920; Stengers 2005; Latour 1995; De Landa 1997) y algunos estudios de caso (obra propia; Vigueras 2016; Philipsz 2010; Geometry Global, 2016; Heuson 2014). Este artículo pretende contribuir con una profundización del concepto de registro sonoro que puede servir a la creación de arte sonoro.

\section{BREVE ESTADO DE LA CUESTIÓN}

\subsection{IdENTIDAD DEL ECOSISTEMA SONORO EN LAS RELACIONES CON LA MÚSICA Y LA SOCIEDAD}

El ecosistema sonoro está determinado por el medio rural o urbano, por las diferentes horas y momentos del día y también por la situación del observador, es decir, de cómo y dónde se sitúa el sujeto que percibe. Implica el reconocimiento del lugar que ocupa el sujeto en la percepción y nos aporta datos sobre cómo se establece su relación con el entorno, de acuerdo a qué circunstancias y situación física.

Cabrelles (2006) considera que los sonidos dan forma al ambiente y definen al mundo que nos rodea como una envoltura espacial y sonora que influye en los habitantes de un ecosistema. Esta complejidad, según Joseph Cerdà (2020), tiene que ver con el desplazamiento del sujeto de un ambiente a otro, por lugares que contienen sonidos específicos y distintos de otros. Podemos afirmar que la identidad sonora del ecosistema es compleja y dinámica, puesto que, miramos frontalmente, pero escuchamos en $360^{\circ}$. El sonido así adquiere una función temporal y espacial en un ambiente sonoro que comprende la totalidad 
de sonidos de un entorno determinado. Según Joseph Cerdà, se trata de una organización espacial y temporal.

La configuración compleja de la identidad de un ecosistema sonoro está determinada por factores objetivos y subjetivos que influencian la percepción.

En la cultura indígena australiana, tanto los lugares específicos y el paisaje como la gente son considerados archivos vivientes de difícil acceso. Los aborígenes australianos caminan el lugar a través del canto, las historias, danzas y rituales como método para reconectar y mantener vivo el lugar, manteniendo a su vez el espíritu y la memoria ancestral. En ese proceso cognitivo a través de la percepción auditiva podemos interpretar los elementos que conforman la identidad de un ecosistema sonoro y traducirlo a través de la música. La música es un ejemplo de superación de la fragmentación entre sujeto y objeto, ya que, para Ramírez Torres (2004), las características de la música están presentes en las relaciones sociales que se ejercen a través de la cadencia musical específica en cada circunstancia comunicativa.

\subsection{ECOSISTEMAS SONOROS CINEMATOGRÁFICOS}

En la película Roma (2018), el director mexicano Alfonso Cuarón utiliza el paisaje sonoro para resaltar algunos aspectos significativos de la identidad mexicana a través de su propia sonoridad anclada en imágenes rodadas en algunos barrios históricos y significativos de la ciudad de México en los años setenta.

El sonido entendido como elemento identitario y relevante en la relación espacial y temporal del sujeto con el entorno, se expresa en la película Lisbon Story (1994) de Wim Wenders, una referencia en la que la presencia de la música, participa como elemento que forma parte del ecosistema sonoro de un lugar.

\subsection{EL REGISTRO SONORO Y ARTE}

En cada registro de campo establecemos una relación entre el ecosistema sonoro, la grabadora y los distintos micrófonos que se pueden utilizar para interactuar, capturar y analizar los sonidos. Esta relación puede establecerse de distintas formas. En cada caso la interferencia del artista que registra el ecosistema sonoro puede ser mayor o menor. En este proceso influye el tipo de tecnología usada, los puntos de toma definidos, la capacidad de los micró- 
fonos, y de la propia grabadora, por citar algunos elementos que interfieren en el registro.

Algunas referencias de procesos de interacción entre la grabación y la creación, utilizando archivos sonoros, la encontramos en artistas como Hidegard Westerkamp (Sound walking), quién define el paseo sonoro como una forma de composición. En el caso de Acoustic mirrors (2013), la presencia del sujeto que graba adopta una actitud que pasa de una menor presencia, a estar más presente, a través de la intervención de la propia grabación.

Un ejemplo relevante es Soundscape \#1 de Nensi Bego y Alice Gussoni (2019) ${ }^{2}$ , una instalación que formó parte de una exposición en MACRO, Museo de Arte Contemporánea de Roma. La estética del sonido, como la del lenguaje, es utilizada para registrar elementos casuales y crear un discurso a través de dicho registro, una especie de Instagram sonoro, destacando el detalle y lo más casual posible, como un juego.

Con la pandemia Covid-19, el proyecto Sound of Tourism (2018) se ve obligado a desarrollar sus planteamientos frente a la incertidumbre que provoca la situación actual y explorar el sonido de calles y espacios que normalmente estarían llenos de turistas para observar los cambios producidos en el ecosistema sonoro por las restricciones derivadas de la pandemia. Basados en sus observaciones han creado una serie de postales sonoras que registran el ambiente sonoro de la ciudad sin turistas, creando Sound postcards from Lisbon (2020) como testimonio del poder que ejerce el paisaje sonoro del turismo en la ciudad, cuya transformación durante el confinamiento trae como consecuencia una serie de sensaciones en un contraste que evoca una cierta intranquilidad, irrealidad o hasta amenaza.

\subsection{Música, SONIDO Y ESPACIO URBANO}

Kleos Cristina Enea (2020) $)^{3}$ es un proyecto que invita a componer piezas sonoras con fragmentos musicales de los lugares originarios de las especies botánicas no autóctonas. El proyecto se desarrolla entre Tabakalera y la Fundación Cristina Enea, en Donostia-San Sebastián.

Soundscapes of the Black Hills (2009) de Jennifer Heuson, son una serie de paisajes sonoros compuestos por registros de grabación en distintos lugares dentro del área de Black Hills en Estados Unidos, cuya zona está compuesta de grupos de montañas que separan un estado de otro (Anderson 2016). Electric 
Museum y Buffalo Growl son algunos de los ejemplos en los que se observa como la artista trabaja a partir de ecosistemas sonoros, a través del registro de lugares significativos, utilizando la grabación de campo para reflexionar sobre los lugares.

Stories of the City: Sailortown (2012) Isobel Anderson y Fionnuala Fagan, son canciones que hablan de la historia del lugar a través del proceso de transformación urbanístico y su problemática, a través de canciones extraídas de entrevistas y grabaciones del ecosistema.

La instalación sonora Polifonía ambulante (2016) del artista francés Félix Blume, en la Fonoteca Nacional de Ciudad de México ${ }^{4}$, hace uso de las sonoridades de los pregones como manifestación popular presente en la identidad mexicana. Los gritos de vendedores ambulantes ${ }^{5}$ son reinterpretados por el artista a través de su obra, resaltando un aspecto particular de la vida cotidiana e interactuando con el público.

\subsection{ACtiVACión deL ESPACIO URBANO A tRAVÉS DEL SONIDO, MÚSiCA Y ARTE}

La obra Lowlands (Philipsz 2010) interviene el espacio a través de la colocación de algunos altavoces y de la acción de cantar debajo de puentes. La artista usa su propia voz para crear instalaciones sonoras aprovechando espacios solitarios o abandonados.

Otra muestra de actitud más presente en la creación a través del sonido y la música, así como la recreación de la identidad del ecosistema sonoro de un lugar, lo encontramos claramente en Activación sonora del espacio público, una serie de talleres llevados a cabo por Fernando Vigueras (2016) ${ }^{6}$. Se trata de una intervención sonora en la Plaza Giordano Bruno, en la Colonia Juárez de la Ciudad de México como parte del Laboratorio Ciudadanía Digital.

Geometry Global (2016) realiza una intervención en calles y espacios públicos de París, interpretando una pieza musical con el fin de enviar un mensaje positivo para el próximo año. Dicha presentación se realiza gracias a que la agencia de comunicación cede las redes de la ciudad para hacerla resonar. 


\section{IDENTIDAD DEL ECOSISTEMA SONORO}

El arte puede ser un medio para establecer relaciones directas con el espacio territorial y a su vez reafirmar las conexiones con los distintos campos del conocimiento. Varios filósofos, entre ellos Manuel De Landa (1997, 2006), Quentin Meillassoux (2006), Markus Gabriel (2015), Gabriel Harman (2015), cuestionan las teorías postmodernas y contribuyen a la construcción de marcos ontológicos más realistas, nuevos realismos o el denominado realismo especulativo. Sin embargo, la vuelta a marcos realistas por parte de estos autores significa un cuestionamiento también del constructivismo. En particular, esta crítica también apunta a las teorías de Niklas Luhman (1998) sobre la idea de construcción social de la realidad (Castillo 2019).

Como afirma Castillo (2019), este marco realista sugiere la idea de que la naturaleza es un sistema abierto en perpetuo devenir. Según la autora, la realidad podría ser entendida como un enjambre de diversos materiales en constante mutación y metamorfosis.

Por un lado, Manuel De Landa es una de las referencias fundamentales en este proceso de crítica a la postmodernidad, al proponer una transversalidad entre la filosofía y otros campos, incluyendo la historia y la cibernética y, en cierta medida, de modo paralelo al pensamiento de Gilles Deleuze, igualmente se expresa de modo interdisciplinar. Para De Landa (2006), el mundo tiene su propia independencia, su morfogénesis, no depende de nuestra mente, ni de nuestra subjetividad. Para De Landa (2012), las especies y los ecosistemas resultan de procesos productores de estructura, idénticos a los que producen en el mundo geológico, el producto histórico de un proceso de consolidación. De cierto modo, esta auto-configuración de los ecosistemas supone una disminución de la importancia del sujeto en la interacción con el mundo, muy presente en el pensamiento de Deleuze.

Por otro lado, estas teorías se alinean con ideas de autores actuales de gran relevancia, tales como pueden ser la ecología de prácticas, la cosmopolítica (Stengers 2005), las teorías actor-red y el concepto de teología política de la naturaleza (Latour 2008; Monbiot 2013).

Desde el análisis de Latour (1995), la política ha convertido la naturaleza en algo ajeno, opuesto a lo cultural y humano, concibiendo lo salvaje como algo que debía quedar fuera de las ciudades para dominarlo o explotarlo hasta llegar a su destrucción, convirtiéndolo en entornos urbanos. Sin embargo, el 
marco realista nos ayuda a comprender la identidad de un ecosistema -y también del ecosistema sonoro- como algo en permanente cambio y transformación, pues estos autores proclaman un reconocimiento de la multiplicidad en el encuentro de diversas prácticas, entre las que se encuentra la denominada rewilding, ideal en la aplicación de los planteamientos de la cosmopolítica de Stenger y que supone una implicación y compromiso en una relación más activa con el medio natural. En este marco, el conocimiento y la técnica, tecnología y tecnociencia, situadas, deben servir para restaurar las relaciones con lo natural.

La cosmopolítica de Stengers propone que los conceptos de lo salvaje y lo domesticado son extremos inalcanzables, y que no se dan en estado puro, pero permiten una composición más o menos congruente con las prácticas contemporáneas y creadoras en un lugar, en las que los conceptos derivados del pragmatismo sirven de guía para la acción. La acción física en el entorno agudiza nuestra sensibilidad y conciencia de lo que sucede a nuestro alrededor, favoreciendo las relaciones con lo no humano. Reconcilia lo natural -lo salvaje- y lo humano, lo social y lo individual. Supone la posibilidad de observar directamente ese entorno natural e intentar una puesta en común que respete las diferencias, más allá de imponer una visión reduccionista y universalista.

Por otro lado, el cuestionamiento de esta oposición dominante en el pensamiento occidental, no implica suprimir la distinción entre lo natural y lo humano, ya que reconocer las interacciones, supone la existencia de una distinción que interactúa, que se co-produce y compone.

Una referencia fundamental para comprender el sonido, como material para la creación en el ámbito de la música contemporánea, experimental y el arte sonoro, es El arte de los sonidos fijados (Chion 1991). De acuerdo a Chion, en las artes plásticas, este material sonoro representa un punto en común y por ello un interesante vínculo entre disciplinas tales como pintura, escultura y dibujo. Igualmente, las ideas fundamentales de Pierre Schaeffer sobre el concepto de música concreta y sus distintas interpretaciones, así como de su desarrollo para la utilización de sonidos grabados como material artístico, nos interesa de manera directa, ya que, nos permite resolver algunos puntos en relación con nuestra metodología durante los procesos en el registro de ecosistemas sonoros y su posterior utilización en la creación artística. 
Desde la perspectiva de las teorías de la complejidad, un ecosistema es un conjunto de sistemas en un sistema abierto en los que se establecen múltiples conexiones. Es una unidad compleja compuesta de partes diversas e interrelacionadas en una constante relación entre caos y orden con dos elementos principales: la interrelación de los elementos y la unidad global constituida por dichos elementos en interrelación (Morin 1981). Según Morin, el ecosistema es una máquina viviente que actúa con base en las interacciones, en una relación de orden- desorden- organización- re-organización.

Apoyándonos en Morin, consideramos que un ecosistema sonoro es un conjunto de manifestaciones sonoras, producidas por las múltiples organizaciones de los seres vivos en constante interacción, que se revelan de manera natural o artificial, según determinados territorios y sus características, así como la organización social y cultural.

Siguiendo los autores mencionados anteriormente, y especialmente a Morin, sugerimos que la identidad sonora de un ecosistema está determinada por sus diversos componentes en la confluencia entre sistemas orgánicos e inorgánicos, como parte de las interacciones con los fenómenos de la naturaleza y de una serie de procesos organizativos en constante emergencia; es el resultado de una serie de interacciones azarosas entre fenómenos múltiples que el ecosistema sonoro desde el azar se da paso a la organización. Desde esta definición, asumimos que el ecosistema participa en los procesos generativos y de la cosmogénesis de manera no consciente. Su identidad se compone de diversos elementos en interacción, en co-organización para crear lo que llamamos identidad del ecosistema sonoro, la cual puede ser determinada por el sujeto como parte del proceso de significación.

Como resultado de los procesos de la toma de conciencia por medio de los sentidos, y de la comunicación con el entorno a través del pensamiento como indica Whitehead (1968), la significación es la forma en que se traducen los fenómenos que se revelan en la naturaleza, para nombrarlos e interactuar con ellos de manera consciente. Para John Dewey (1948), el pensamiento y la razón consisten en procesos utilizados de manera predeterminada para adaptar lo confuso e indeterminado, a lo regular y estable, y viceversa.

Para este autor, la significación es un método de acción, y la manera de usar cosas como medios para conseguir un fin determinado, y afirma que el método es general, aunque las cosas a las que se apliquen sean particulares. En el 
método está la sujeción de lo observado, es decir, lo subjetivo en cuanto a las relaciones con los fenómenos.

\section{ANÁLISIS DE UN CASO DE ESTUDIO}

Como parte del desarrollo práctico y teórico de mi tesis, propongo un análisis de registros sonoros y una tabla de actitudes artísticas respecto a los casos artísticos analizados. Esa base de datos contiene más de 1000 registros de audio desde 2015 a 2017, realizados en España, México y Francia. Estos archivos se han utilizado para experimentar a través de la creación sonora y audiovisual, como registro de acciones e intervenciones que he realizado en espacios locales como en Bilbao, y para un análisis objetivo a partir de una selección, experimentando y explorando las distintas formas de creación artística a través del sonido.

\begin{tabular}{|c|c|c|c|c|c|c|}
\hline \multicolumn{7}{|c|}{ Anexo 1} \\
\hline \multicolumn{7}{|c|}{ Clasificación de registros por año desde 2005} \\
\hline No. & Nombre y tipo de archivo & Duración & Lugar & Descripción & Primera categoría & $\begin{array}{l}\text { Nivel de } \\
\text { actividad }\end{array}$ \\
\hline & & & & & & 2005 \\
\hline 1 & Alumnos 2005.WAV (estéreo) & 18:18 & Vitoria-Gasteiz & $\begin{array}{l}\text { Clase de guitarra en el Colegio Urkide } \\
\text { Eskola. Alumnas cantando }\end{array}$ & $\begin{array}{l}\text { Ambiente interior diurno en un aula: } \\
\text { enseñanza musical }\end{array}$ & \\
\hline 2 & Track 1.WAV (estéreo) & $0: 47$ & Vitoria-Gasteiz & Manifestación en apoyo a una ocupación & \begin{tabular}{|l|} 
Ambiente esterior diurno: \\
manifestaciones musicales y sociales
\end{tabular} & \\
\hline 3 & Track 3.WAV (estéreo) & 0.29 & Vitoria-Gasteiz & Conversación & $\begin{array}{l}\text { Ambiente interior de una casa } \\
\text { conversaciones }\end{array}$ & \\
\hline 4 & Track 7.WAV (estéreo) & $0: 14$ & Vitoria-Gasteiz & Documental huelga general del 3 de Marzo & Estractos sonoros televisivos & \\
\hline 5 & Track 9.WAV (estéreo) & $0: 39$ & Vitoria-Gasteiz & Manifestación en apoyo a una ocupación & Manifestaciones sociales & \\
\hline 6 & Track 13.WAV (estéreo) & 0.25 & Vitoria-Gasteiz & $\begin{array}{l}\text { Yo tocando la trompeta dentro de una } \\
\text { habitacion de un espacio ocupado }\end{array}$ & Autograbación musical & \\
\hline & & & & & & 2006 \\
\hline 7 & 02 Pista 2.wav (estéreo) & $0: 34$ & Barcelona & $\begin{array}{l}\text { Dentro del vagón del metro voz fenemenina } \\
\text { anunciando estacion }\end{array}$ & Trasnporte público: voz-máquina & \\
\hline
\end{tabular}

Fig. 1: Detalle de la base de datos y clasificación de archivos sonoros desde 2005, fotografía de Jaime A. Cornelio Yacaman, 2020.

Partiendo de la definición de ecosistema sonoro, desarrollo una serie de criterios de análisis e identificación de elementos fundamentales de identidad en 
los ecosistemas sonoros concretos. Ello condujo a la creación de una tipología, a partir del análisis de la actitud de los artistas en relación con el ecosistema (durante la toma de registros o en su modo de usar el ecosistema en la obra plástica) realicé una tipología de actitudes en relación con el ecosistema.

Desde el punto de vista de la actitud del artista en relación con el ecosistema sonoro, se diferencian tres actitudes: más presente, menos presente e híbrida. Lo que me condujo a observar que la identidad del ecosistema sonoro es utilizada por distintos artistas y de múltiples maneras, de modo más o menos presente en cuanto al nivel de relación que se establece entre el sujeto y el entorno. A estas relaciones las denomino actitudes, siendo ellas más directas e indirectas según los casos, ya sea para transformar el propio ecosistema, para traducirlo o simplemente para reproducirlo.

TABla de a Actitud artística en RELACión CON EL ECOSistema

\begin{tabular}{|l|l|l|l|}
\hline $\begin{array}{l}\text { Actitud artística en } \\
\text { relación con el ecosistema }\end{array}$ & $\begin{array}{l}\text { Naturaleza y } \\
\text { manipulación del propio } \\
\text { ecosistema }\end{array}$ & Registro & Creación \\
\hline Actitud más presente & & & \\
\hline Actitud híbrida & & & \\
\hline Actitud menos presente & & & \\
\hline
\end{tabular}

El grado de incidencia por parte del sujeto es más presente si lo manipula y modifica. Una forma de relación menos presente se contempla en los casos en el que se utiliza el registro natural o paisaje sonoro de un lugar, disminuyendo la participación activa y directa por parte de sujeto. También se observa que existen casos en que ambas actitudes aplicadas en conjunción, dan como resultado una serie de expresiones híbridas en las que se pueden encontrar posibles formas de trabajar con el material sonoro para la creación artística.

Durante todo ese proceso he podido contabilizar 459 archivos utilizables hasta 2016, y cerca de casi un total de 1000 hasta 2017, registros sonoros in situ en diferentes lugares por espacios de tiempo, desde un minuto como mínimo y un 
máximo de hora y media, trazando determinadas rutas y trayectos. De estos recorridos he podido extraer una serie de sonidos que permiten identificar elementos propios de un espacio, tiempo y lugar en movimiento.

Muchos de estos registros se han hecho con especial atención en rutas que conforman el espacio urbano, el transporte público y todo lo que sucede en él en distintos espacios de tiempo. Como recursos previos conté con 109 archivos grabados y contabilizados desde el año 2005 hasta 2014 en México, Portugal, Cuba, París y también en distintas zonas y barrios de Bilbao y Vitoria-Gasteiz. Este proceso permitió desarrollar un repositorio de registros sonoros, editados y alojados en varias páginas de Soundcloud. La primera se titula Identidades sonoro-espaciales $(2016)^{7}$, con 52 registros tanto de entornos como recorridos cantados y en silencio, algunas experimentaciones, así como las creaciones sonoras Identidades sonoro-espaciales Serie 1 (ciudad).

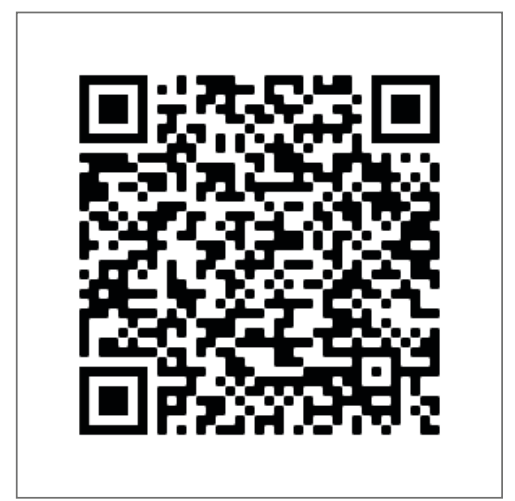

Recorridos cantados: https://cutt.ly/blU2uT6

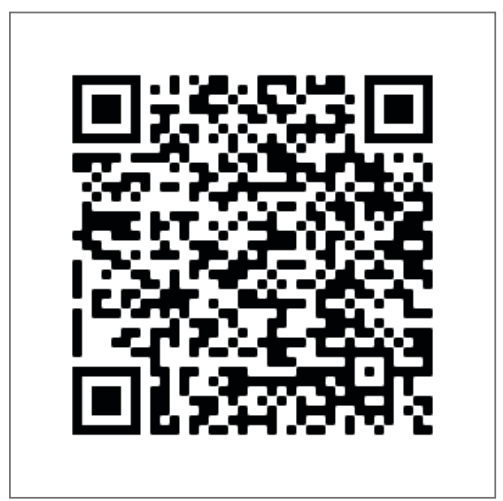

Recorridos sonoros: https://cutt.ly/0IU0V40

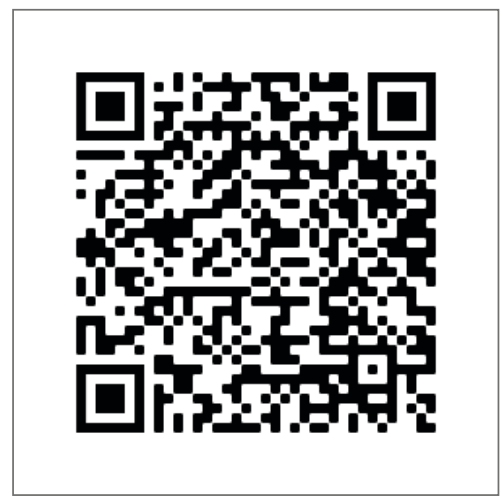

Identidades sonoro-espaciales:

https://cutt.ly/hlU2Ehg 
Identidad de ecosistemas sonoros $(2020)^{8}$ presenta las primeras conclusiones y muestra 27 audios que corresponden a una selección de archivos analizados y traducidos en términos musicales y visuales a partir de una lectura del espectrograma y los datos de frecuencia y pulsaciones (cepstrum).

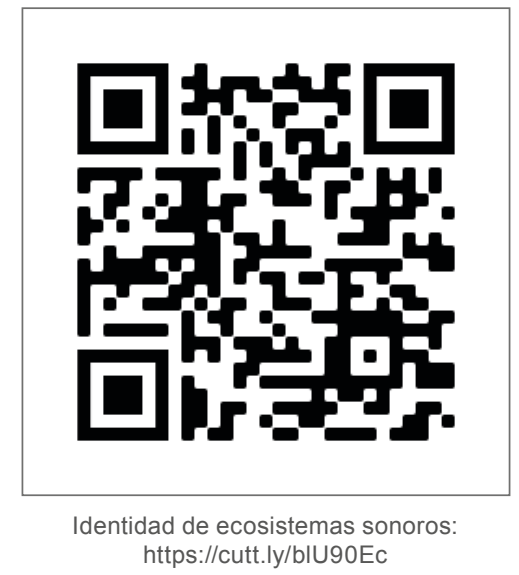

Por lo que actualmente, estas tres plataformas, cuentan con un total de 79 audios. Estos registros representan un tipo de relación que se establece con el ecosistema y a su vez, con el propio registro sonoro y las posibilidades en el ámbito de la creación artística contemporánea.

A través del arte, las actitudes menos presentes se hacen más presentes, y, conjuntando ambos casos, el resultado son formas de relación híbridas de las que surgen nuevas formas de creación. La propuesta de una tipología de actitudes y el grado de exploración de la identidad del ecosistema sonoro puede servir como base para la creación artística en esa confluencia entre sonido, música, arte y sociedad, en cuanto a la exploración y la activación de espacios públicos, por ejemplo, en la conformación de la identidad sonora territorial.

\section{Referencias bibliográficas}

Anderson, Isobel. 2016. "Soundmapping beyond the grid: Alternative cartographies of sound". Journal of Sonic Studies 11. https://www.researchcatalogue.net/view/234645/234646

Cabrelles Sagredo, María Soledad. 2006. "El paisaje sonoro: 'Una experiencia basada en la percepción del entorno acústico cotidiano'”. Revista de Folklore 302: 49-56. http://www. cervantesvirtual.com/obra-visor/el-paisaje-sonoro-una-experiencia-basada-en-la-percepcion-del-entorno-acustico-cotidiano/html/ 
Castillo Villapudua, Karla. 2019. "Claves teóricas en Manuel De Landa: De la ontología deleuziana, los ensamblajes, emergentismo y la historia no lineal”. Andamios (16)40: 229-50. http://dx.doi.org/10.29092/uacm.v16i40.705

Cerdà i Ferré, Josep, Josep Manuel Berenguer Alarcón \& Martí Ruiz Carulla. 2020. "Principios básicos del paisaje sonoro" Curso Mooc Universitat de Barcelona. https://es.coursera.org/ learn/introduccion-arte-sonoro

Chion, Michel. 1991. El arte de los sonidos fijados. Traducción, Carmen Pardo. Cuenca: Centro de Creación Experimental

De Landa, Manuel. (1997) 2012. Mil años de historia no lineal. Barcelona: Gedisa

De Landa, Manuel. 2006. A new philosophy of society: Assemblage theory and social complexity. London: Continuum

De Landa, Manuel. 2008. "The virtual breeding of sound”. En Sound unbound: Sampling digital music and culture, edited by Paul D. Miller, Cambridge MA: MIT. https://doi.org/10.7551/ mitpress/7723.003.0021

Deleuze, Gilles \& Felix Guattari. (1972) 2002. Mil Mesetas: Capitalismo y esquizofrenia. Traducción de José Vázquez Pérez, con la colaboración de Umbelina Larraceleta. Valencia: Pre-textos

Dewey, John. (1925) 1948. La experiencia y la naturaleza. Prólogo y versión española de José Gaos. Ciudad de México: Fondo de Cultura Económica

Gabriel, Markus. 2015. Por qué el mundo no existe. Traducción de Juanmari Madariaga. Barcelona: Pasado y Presente

Geometry Global. 2016. "Paris résonne: Meilleurs vœux 2016 de Geometry Global Paris". Happening musical. Vídeo de Youtube, 1:08, 22 ene. https://www.youtube.com/watch?v=dDBuGbvfwy4

Harman, Gabriel. (2010) 2015. Hacia el realismo especulativo: Ensayos y conferencias. Traducción, Claudio Iglesias; edición al cuidado de Florencio Noceti. Buenos Aires: Caja Negra

James, Diana. 2015. “Tjukurpa time”. En Long history, deep time: Deepening histories of place, edited by Ann McGrath \& Mary Anne Jebb, 33-45. Canberra: Australian National University. https://doi:10.22459/LHDT.05.2015

Latour, Bruno. 1995. “¿Tienen historia los objetos? El encuentro de Pasteur y Whitehead en un baño de ácido láctico". Traducido por Javier Echeverría. Isegoría 12: 92-109. doi. org/10.3989/isegoria.1995.i12.242

Latour, Bruno. 2008. Reensamblar lo social: Una introducción a la teoría del actor-red. Traducción, Gabriel Zadunaisky. Buenos Aires: Manantial

Meillassoux, Quentin. (2006) 2015. Después de la finitud: Ensayo sobre la necesidad de la contingencia. Prefacio, Alain Badiou; traducción, Margarita Martínez; edición al cuidado de Florencio Noceti. Buenos Aires: Caja Negra

Monbiot, George 2013. Feral: Searching for enchantment on the frontiers of rewilding. London: Penguin

Morin, Edgar. (1981) 2010. El método, I. La naturaleza de la naturaleza. Traducción de Ana Sánchez en colaboración con Dora Sánchez García. Madrid: Cátedra

Philipsz, Susan. 2010. "'Lowlands': Susan Philipsz, Turner Art Prize Winner 2010”. Vídeo de YouTube, 8:25. 18 mayo. https://www.youtube.com/watch?v=UWeKzTDi-OA 
Ramírez Torres, Juan Luis. 2004. "El sonido numinoso: Música ritual y biología". Convergencia (36): 81-97. https://convergencia.uaemex.mx/article/view/1527/1165

Rodríguez Pacheco, Darnika. 2018. "Los escenarios reales de 'Roma' que puedes encontrar en México". El País Verne, 17 dic. https://verne.elpais.com/verne/2018/12/17/ mexico/1545062725_147190.html

Sánchez-Fuarros, Iñigo \& Daniel André Fernandes Paiva. 2020. “¿A qué suena una ciudad turística sin turistas?” The Conversation, 23 jun. https://theconversation.com/a-que-suenauna-ciudad-turistica-sin-turistas-140351

Sánchez-Fuarros, Iñigo \& Salwa El-Shawan Castelo-Branco. 2020. "Sounds of sonification map". Sound of Tourism (web). http://www.soundsoftourism.pt/mapping-sonic-turistification/

Stengers, Isabelle. 2005. "The cosmopolitical proposal". En Making things public: Atmospheres of democracy, exhibition at the ZKM, Center for Art and Media in Karlsruhe, 20.03- 03.10, edited by Bruno Latour \& Peter Weibel. Cambridge MA: MIT

Westerkamp, Hildegard. (1974) 2013. "Hildegard Westerkamp: 'Soundwalking'. Posted by Kamen Nedev. Acoustic Mirror (blog), 26 jun. Publ. orig.: Sound Heritage 3(4): 18. http:// acousticmirror.tumblr.com/post/53666735092/hildegard-westerkamp-soundwalking

Whitehead, Alfred North. (1920) 1968. El concepto de la naturaleza. Versión española de Jesús Díaz. Madrid: Gredos

\section{Notas}

${ }^{1}$ Enclave de Ciencia (RAE-FECYT), s.v. "ecosistema" https://enclavedeciencia.rae.es/diccionarios/dlect/search/zones?zones\%5B\%5D=header:ecosistema

${ }^{2}$ Nensi Bego \& Alice Gussoni: "Soundscape \#1 Rome" (2019). 67 tracks audio. https://soundcloud.com/user-822407203

3 "Descarga Kleos Cristina Enea" by Cultura y Ciudadanía - Cristina Enea / 30-10-2020. https:// gardenatlas.net/garden/kleos-cristina-enea/post/28526? filter=3

4 "Invita la Fonoteca Nacional a jugar con los sonidos de la ciudad en la exposición Polifonía ambulante". Secretaría de Cultura Gobierno de México, 10 jun. 2016. http://www.gob.mx/ cultura/prensa/invita-la-fonoteca-nacional-a-jugar-con-los-sonidos-de-la-ciudad-en-la-exposicion-polifonia-ambulante?state=published

${ }^{5}$ Félix Blume: "Los gritos de México" (2015). 1 track audio, 29:11. https://soundcloud.com/felixblume/losgritosdemexico

${ }^{6}$ Vigueras, Fernando. "Activación sonora del espacio público: Plaza Giordano Bruno_ CDMX". Laboratorio de Ciudadanía Digital-MotorLab. Vídeo de Youtube, 2:30. 15 sept. 2016. https://www.youtube.com/watch?v=HNjzXApF3As\&feature=youtu.be

7 Jaime Alejandro Cornelio Yacaman: "Identidades sonoro-espaciales" (2016). 73 tracks audio. https://soundcloud.com/identidades-sonoro-espaciales-2016

8 Jaime Alejandro Cornelio Yacaman: “Identidad de ecosistemas sonoros" (2020). 58 tracks audio. https://soundcloud.com/user-360049681 